\title{
NOUVELLE
}

\section{La thérapie photodynamique}

\section{Application au traitement chirurgical des glioblastomes}

Clément Dupont $^{1}$, Maximilien Vermandel ${ }^{1,2}$, Nicolas Reyns ${ }^{1,2}$, Serge Mordon ${ }^{1}$

${ }^{1}$ Université de Lille, Inserm, CHU de Lille, U1189 - ONCO-THAI - Thérapies laser assistées par l'image pour l'oncologie, 59000 Lille, France.

${ }^{2}$ Service de neurochirurgie, CHU de Lille, 59000 Lille, France. maximilien.vermandel@chru-lille.fr
> Les gliomes sont des tumeurs cérébrales primitives classés du grade I au grade IV en fonction du degré de leur malignité [1], et selon la dernière classification de l'Organisation mondiale de la santé (OMS). Parmi les gliomes de haut grade, le glioblastome (GBM) est la tumeur cérébrale primitive maligne la plus fréquente chez l'adulte avec une incidence moyenne de 4 cas par an pour 100000 habitants en France, et une médiane de survie de 15 mois avec le traitement standard [2]. Le GBM se caractérise par la capacité d'infiltration des cellules tumorales dans le parenchyme cérébral et $(\rightarrow)$ Voir la Nouvelle de par sa grande hété- M. Verreault et al., $\mathrm{m} / \mathrm{s}$ rogénéité [3] $(\rightarrow)$. $n^{\circ} 10$, octobre 2012, Aucun facteur étio- page 813 logique externe n'a, pour le moment, été rapporté, à l'exception d'une exposition à des rayonnements ionisants utilisés dans le cadre de précédentes thérapies cérébrales.

\section{Le standard de soin}

Un standard de soin est actuellement proposé à l'ensemble des patients nouvellement diagnostiqués [4]. II se compose d'une résection chirurgicale lorsqu'elle est possible, suivie d'un traitement par une radio-chimiothérapie concomitante suivie d'une chimiothérapie adjuvante.

La qualité de l'exérèse demeure un facteur pronostic important, qu'il s'agisse de la survie sans progression ou de la survie globale. Cette dernière est plus importante pour des patients ayant bénéficié d'une résection complète (16,9 mois de médiane) que pour ceux dont la résection a été incomplète (11,8 mois, $p<0,0001)$ [5].

La radio-chimiothérapie adjuvante améliore le contrôle de la maladie par son effet cytotoxique sur les cellules tumorales résiduelles, mais ce protocole, appliqué depuis 2005, n'a permis une augmentation de la médiane de survie que de 12,1 mois à 14,6 mois [6] .

\section{Technique chirurgicale}

Une technique chirurgicale recourant à une molécule fluorescente a permis une augmentation de la médiane de survie de 2 mois sans progression [7]. Un agent médicamenteux (I'acide 5-aminolévulinique, 5-ALA) est en effet administré au patient quelques heures avant l'intervention chirurgicale. Une fois métabolisée, cette molécule induit la surproduction d'une seconde molécule photosensible dans les cellules: la protoporphyrine IX (PpIX). Les cellules tumorales ne peuvent éliminer cet excès de PpIX ainsi apporté, en raison d'un déficit enzymatique; un différentiel de concentration va alors se créer entre cellules saines et cellules tumorales. Lorsque la cavité opératoire est exposée à la lumière bleue (375 - 440 nm), la PpIX excitée émet une lumière de fluorescence rouge $(635 \mathrm{~nm})$ qui guide le geste chirurgical permettant de distinguer le tissu tumoral du tissu sain, améliorant ainsi la qualité de l'intervention. On parle de chirurgie guidée par fluorescence.
Ce traitement chirurgical améliore la survie des patients, mais ne permet pas leur guérison. En effet, la composition cellulaire du GBM et, plus particulièrement, la présence de cellules souches cancéreuses auto-renouvelables contribuent à la récidive. Les chimiothérapies conventionnelles ne parviennent pas, non plus, à apporter une réponse efficace pour le traitement du GBM, en raison d'une mauvaise pénétration des produits au travers de la barrière hémato-encéphalique.

Même si la chirurgie est jugée complète (ne laissant apparaître aucune fluorescence visible ou aucun signal par IRM [imagerie par résonnance magnétique], qui seraient la signature de reliquats tumoraux), la récidive est inéluctable et apparaît dans $85 \%$ des cas dans les marges de la cavité opératoire [7]. Le caractère infiltrant du glioblastome dans le parenchyme adjacent explique en grande partie cet échec thérapeutique. Dans ce contexte, la thérapie photodynamique peropératoire a été développée afin de traiter les berges de la cavité opératoire et ainsi améliorer le contrôle local de la tumeur.

\section{La thérapie photodynamique}

La thérapie photodynamique (ou PDT) repose sur la réaction photochimique induite par l'exposition à la lumière laser d'une molécule photosensibilisante, ou d'un photosensibilisateur (PS), avec l'oxygène naturellement présent. La combinaison de ces trois composants (molécule photosensibilisante, oxygène, lumière laser) conduit à la formation d'espèces 


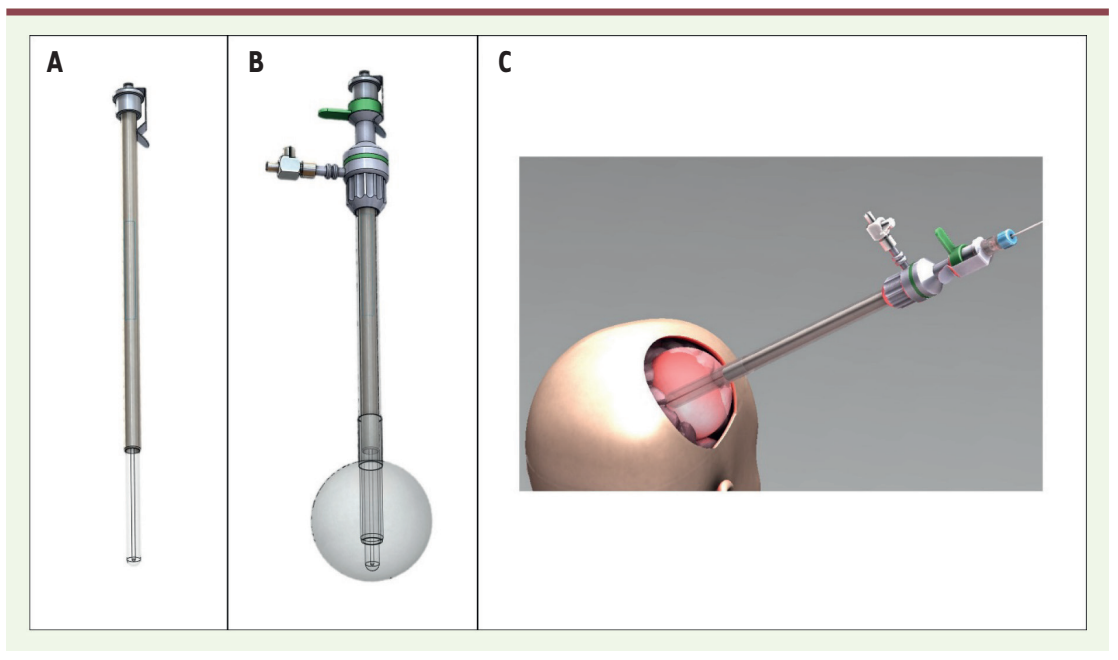

Figure 1. Dispositif original d'illumination. A. Une vue en 3 dimensions du guide lumineux, B. du dispositif complet (ballonnet et guide lumineux). C. Illustration de l'implantation du dispositif dans la cavité opératoire.

réactives de l'oxygène (comme l'oxygène singulet) cytotoxiques.

La séquence d'un traitement PDT commence par l'administration du PS par voie topique, orale ou par injection intraveineuse. Dans le cadre du traitement du GBM par PDT, le PS est la protoporphyrine IX (PpIX), molécule précédemment décrite dans la chirurgie guidée par fluorescence. Le processus moléculaire est similaire à celui utilisé en résection guidée par fluorescence, le principe étant fondé sur les mêmes métabolites. Lorsque illuminée par la lumière rouge $(635 \mathrm{~nm})$, la PpIX se trouve dans un état excité. Elle réagit alors avec l'oxygène présent dans les cellules. Cette réaction photochimique conduit à une destruction directe des cellules tumorales, due à la création d'oxygène singulet, mais aussi indirecte par la stimulation temporaire du système immunitaire.

\section{La PDT lors de la prise en charge chirurgicale}

L'idée principale est de bénéficier de la présence de la PpIX, déjà administrée pour la chirurgie guidée par fluorescence et donc présente dans les cellules tumorales résiduelles, et de la source lumineuse placée au plus proche de la cible thérapeutique, afin d'augmenter le volume de tissu illuminé. La délivrance du traitement PDT à la fin de la résection chirurgicale permet d'accéder directement aux tissus environnant la cavité opératoire, région où la probabilité d'une récidive est la plus élevée. $\varepsilon$ n traitant ces berges tumorales, l'objectif est donc de diminuer le risque de récidive et, par conséquent, d'augmenter la survie sans progression des patients tout en préservant leur qualité de vie.

\section{Un dispositif original d'illumination}

Un dispositif d'illumination couplé à un modèle dosimétrique a été développé (Figure 1). Il est composé de deux parties : 1) un ballonnet (commercialisé par B-Braun ${ }^{\circledR}$ Aesculap ${ }^{\circledR}$, Tuttlingen, Allemagne) et 2) un guide lumineux transparent maintenant la fibre optique au centre du ballon, développé au sein de notre laboratoire OncoThAl. Afin d'améliorer l'homogénéité de la distribution de la lumière, ce dernier est gonflé à l'aide d'une solution diffusante (solution intralipidique à la concentration de $0,1 \%)$. L'émission de lumière par ce dispositif a été mesurée lors d'expérimentations réalisées ex-vivo. Une fonction de transfert a été modélisée afin de déterminer la durée d'illumination en fonction du volume de la cavité opératoire. En effet, la durée d'illumination est déterminée par la quantité de liquide injectée dans le ballon afin de délivrer l'énergie suffisante à un effet thérapeutique dans les berges.

\section{INDYGO : une étude pilote de PDT}

Le dispositif lumineux ayant été caractérisé, évalué et validé, une étude clinique de faisabilité a été mise en place afin d'évaluer la toxicité de cette technique. L'étude pilote INDYGO (intraoperative photodynamic therapy for glioblastomas) est actuellement en cours (Figure 2) [8]. L'objectif principal de cette étude est d'évaluer la faisabilité de réaliser la PDT peropératoire au cours de la chirurgie d'exérèse du GBM nouvellement diagnostiqué, en l'absence de toxicité immédiate. Les objectifs secondaires sont l'évaluation de la survie sans progression, la survie globale, la réponse au traitement évaluée en imagerie tous les 3 mois, la toxicité au cours du temps, le suivi des événements indésirables potentiellement graves et de la qualité de vie des patients. Dix patients ont été recrutés pour cette phase. Des études cliniques de phases supérieures sont envisagées avec un nombre plus important de sujets par l'inclusion de plusieurs centres recruteurs.

Une étude ancillaire à l'étude INDYGO réalisée sur une partie des patients recrutés a pour objectif d'explorer la réponse immunitaire des patients traités par la recherche de biomarqueurs possiblement induits par le traitement PDT. À l'avenir, une synergie entre un traitement PDT couplé à une immunothérapie ciblée pourrait répondre de façon plus globale au traitement du GBM.

\section{Conclusion}

Le glioblastome est la tumeur cérébrale primitive la plus fréquente. Les thérapies conventionnelles actuelles ne permettent pas d'éviter la récidive et la progression de la maladie. La PDT pourrait devenir une modalité adjuvante et contribuer à améliorer la prise en charge des patients. Un 


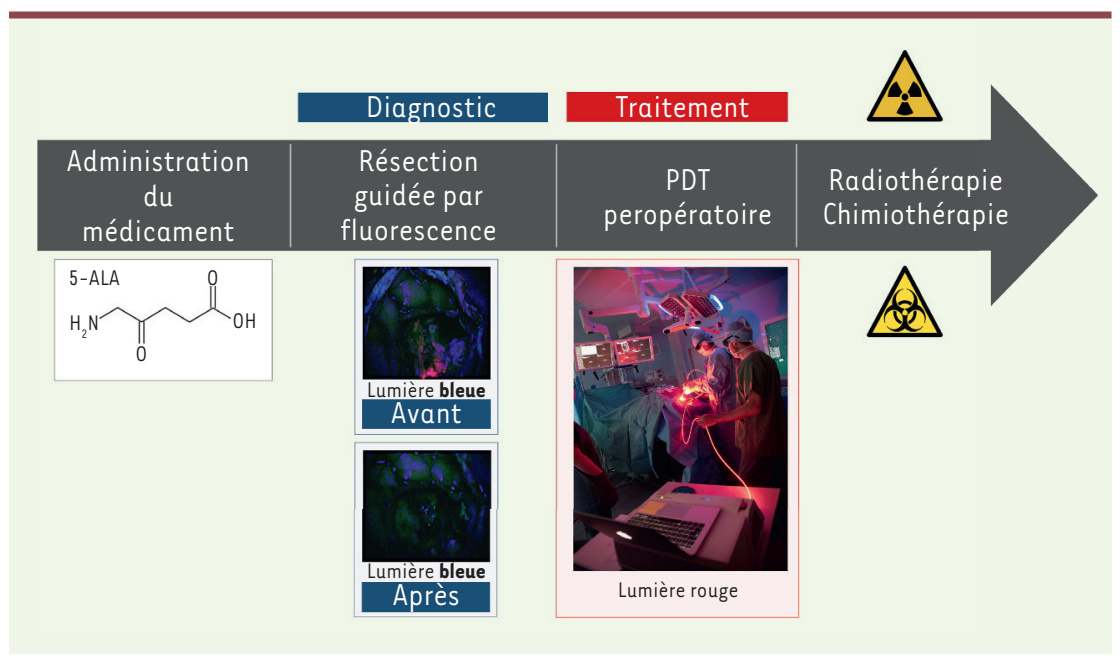

Figure 2. L'étude INDYGO (intraoperative photodynamic therapy for giyoblastomas). L'essai INDYGO est une étude pilote de faisabilité d'une thérapie photodynamique peropératoire du glioblastome. Le protocole de soin inclut le traitement par thérapie photodynamique (PDT) peropératoire en fin de résection. Un agent médicamenteux (I'acide 5-aminolévulinique, 5-ALA) est administré au patient quelques heures avant l'intervention chirurgicale. Une fois métabolisée, cette molécule va induire la surproduction d'une seconde molécule photosensible dans les cellules : la protoporphyrine IX (PpIX). Cependant, les cellules tumorales ne pouvant pas éliminer cet excès de $\mathrm{PpIX}$ en raison d'un déficit enzymatique, un différentiel de concentration va se créer entre les cellules saines et tumorales. Une fois la chirurgie d'exérèse terminée, cette molécule excitée par une lumière rouge $(635 \mathrm{~nm})$ va réagir avec l'oxygène présent dans les cellules et produire de l'oxygène singulet qui sera responsable de la destruction directe des cellules tumorales.

dispositif et sa dosimétrie ont été développés afin d'apporter une solution technique à la mise en place d'un traitement PDT peropératoire. L'étude clinique INDYGO évaluera la faisabilité et la toxicité de la PDT peropératoire au cours de la chirurgie
Photodynamic therapy for the treatment of glioblastoma in neurosurgery

\section{LIENS D'INTÉRÊT}

Les auteurs déclarent n'avoir aucun lien d'intérêt concernant les données publiées dans cet article.

\section{RÉFÉRENCES}

1. Louis DN, Perry A, Reifenberger G, et al. The 2016 world health organization classification of tumors of the central nervous system: a summary. Acta Neuropathol 2016; $131: 803-20$.

2. Stummer W, Pichimeier U, Menel T, et al. Fluorescence-guided surgery with 5 -aminolevulinic acid for resection of malignant glioma: a randomised controlled multicentre phase III trial. Lancet Oncol $2006 ; 7: 392-401$.

3. Verreault $M$, Delattre Jy, Idbailh A. Les tumeurs gliales diffuses de l'adulte - Hétérogénéité génétique et implications thérapeutiques. Med Sci (Paris) 2012; $28: 813-6$.

4. Stupp R, Brada M, van den Bent MJ, et al. Highgrade glioma: ESMO clinical practice guidelines for diagnosis, treatment and follow-up. Ann Oncol 2014 ; 25 : iii93-101.

5. Stummer W, Reulen HJ, Meinel T, et al. Extent of resection and survival in glioblastoma multiforme: identification of and adjustment for bias. Neurosurgery $2008 ; 62: 564-76$

6. Stupp R, Hegi ME, Mason WP, et al. Effects of radiotherapy with concomitant and adjuvant temozolomide versus radiotherapy alone on survival in glioblastoma in a randomised phase III study: 5 -year analysis of the EORTC-NCIC trial. Lancet Oncol $2009 ; 10: 459-66$.

7. Leroy HA, Vermandel M, Lejeune JP, et al. Fluorescence

\section{Bon de commande à retourner à EDP Sciences, 17 avenue du Hoggar, 91944 Les Ulis Cedex}

Tél. : 0149856069 - Fax : 0149850345 - E-mail : francois.flori@edpsciences.org

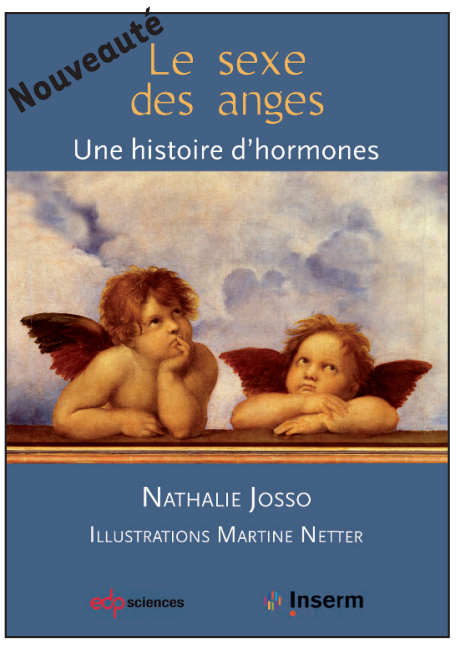

NOM :

Prénom :

Adresse :

Code postal :

Ville :

Pays :

Fonction :

Je souhaite recevoir l'ouvrage

Le sexe des anges : $20 €+3 €$ de port $=23 €$ TTC

en

exemplaire, soit un total de

Par chèque, à l'ordre de EDP Sciences

Par carte bancaire :

$\square$ Visa

Carte $n^{\circ}$

Date d'expiration :

$\mathrm{N}^{\circ}$ de contrôle au dos de la carte : guided resection and glioblastoma in 2015: A review. Lasers Surg Med $2015 ; 47$ : 441-51.
8. Dupont C, Vermandel M, Leroy HA, et al. Intraoperative photodynamic therapy for glioblastomas: study protocol for a phase I clinical trial. Neurosurgery 2018 : nyy324.
tiqué. Ces travaux pionniers de recherche
ont permis le transfert des connaissances de la PDT vers la clinique et la mise en place des premiers traitements photodynamiques en neurochirurgie en France. $\diamond$ 